\title{
Sailing Through a Storm: The Importance of Dialogue in Student Partnerships
}

\author{
Kirsty Macfarlane, Jarah Dennison, Pamela Delly, and *Damir Mitric, Learning Futures, La \\ Trobe University, Australia
}

Contact: d.mitric@latrobe.edu.au

\section{INTRODUCTION}

In late 2016, we-Damir Mitric and Pam Delly-were tasked by the Director of Learning and Teaching at La Trobe University (LTU) in Australia with designing a new operational model for the Peer Learning Adviser (PLA) program, which had traditionally offered one-on-one, peerto-peer support through a centrally located drop-in service. The new model conceptualized student learning as the what of academic development work and staff learning as the how. In this system, academic developers supported student learning by supporting academics as they engaged in practice.

Research indicated that LTU cohorts did not exhibit help-seeking behaviours, so the principal change involved embedding PLA support in curriculum through design and instructional effort. It also involved challenging and transforming the institutional culture and understanding of the role of students in the learning process-particularly in relation to systems that encourage students to engage with each other. To redesign and deliver the PLA program in 2017, Damir and Pam partnered with co-authors Kirsty Macfarlane and Jarah Dennison, who were LTU students and Peer Learning Leaders (PLL). PLLs managed the daily operations of the PLAs and provided academic support for students, while PLAs were solely responsible for providing academic support. We believed that such a partnership had the potential to challenge previous conceptualizations of the program.

The following is our collective attempt-staff- and student-centric, both in terms of outcomes and reporting - to unpack the complexities of our collaborative endeavour in 2017. We juxtapose our respective experiences of navigating the "normative hierarchical university paradigm" (Mercer-Mapstone et al., 2017, p. 18) to present a more collaborative and balanced discussion of our partnership. We reflect on our "way of doing things" (Healey, Flint, \& Harrington, 2014, p. 12) so that the partnership process is more visible, particularly in relation to the challenges and negative outcomes.

An ethos of reciprocity (Matthews, 2017) influenced our thinking and practice, and we were acutely aware of the complexities involved in real-life exchanges between staff and 
students. We discussed power openly throughout our collaboration, and here we speak about its function as equal co-authors of our empirical story. We are frank about the challenges that we faced and do not shy away from discussing failures, as well as lessons learned. We hope that this will help others to critically analyse and reflect on their own practice and, in the process, fully explore the transformative power of student partnerships for individuals and their institutions.

\section{THE CROSSING}

\section{Damir Mitric and Pam Delly}

Cook-Sather's (2014) work on partnership as a threshold concept in academic development-both troublesome and transformative-heavily influenced our redesigned PLA program. As academic developers, we saw an opportunity to transform curriculum into a creative space where fellow academics and students could reimagine the teaching and learning process and question their respective roles in it. To achieve this, we invited subject coordinators to embed the PLA service (through PLA-led workshops) in their teaching cycle, contrary to previous norms of practice. Faculty academics, academic developers, and current students collaborated to find the best way to embed peer-to-peer support into the curriculum -essentially performing a small-scale curriculum review and redesign. Once we agreed how to strategically integrate PLA support into the syllabus, PLAs delivered weekly subject-specific workshops. This significantly challenged the previous patriarchal understanding of students' roles in teaching and learning.

These transformations proved to be troublesome for some academic colleagues in our central unit-particularly those with an Academic Language and Learning (ALL) background, who struggled to make sense of their new hybridized identities (Percy, 2014). Previous dominant academic discourses at our university framed the PLAs as young, inexperienced, and vulnerable individuals in need of ongoing support and protection. In practice, this meant that PLAs performed specific and limited tasks under supervision of academic staff who protected both the quality of the service as well as students' wellbeing. For staff with an ALL background (whose expertise and student-centred work focused on developing academic literacies), PLA's excursion into the curriculum was "counter intuitive or even intellectually absurd at face value" (Meyer \& Land, 2006, p. 4). It challenged their long-held epistemological assumptions and practices grounded in the necessity of one-on-one student support.

While our new student partnership model introduced the possibility of new ways of working in contemporary higher education, it was also more problematic than we had anticipated. The new model challenged the traditional role of students in higher education, leading some colleagues to feel threatened. Perhaps the most difficult part of this process was realizing they felt betrayed, as they saw us as complicit in one of the many neoliberal attacks on the nature and organization of higher education. In this interpretation, students were perceived as being exploited as cheap labour, replacing the role of academics.

While we felt confident that our attempt to shift the nature of our relationship with the PLAs was both timely and appropriate-that it would be productively disruptive-we understood our colleagues' critique of the neoliberal university. We were fully aware of the effects massification, marketization, and managerialism have had on our practices and 
identities. We realized that the policy drivers shaping higher education today were inescapable. However, we also believed that we could subvert these dominant discourses. While we couldn't escape the matrix of the fully-fledged corporate university, we could use its own systems and logic to resist it. In using a business model to frame our partnership, we utilized the language of the dominant discourse in order to subvert it. Subversion, for us, meant staying competitive with the expanding synchronous, online, tutoring services in order to protect our colleagues' jobs.

We do not subscribe to the business approach to education, but we learned to stay relevant by reporting on the impact of our program in language that senior management understood. This language was statistical and reported service and financial cost efficiencies. We agree with Matthews (2017) that, because of their dialogic nature, student partnerships provide a "counter-narrative to current neoliberal agendas" (p. 1), but we also note that in practice the story is more complicated. Student partnerships open a Pandora's box of what it means to be an academic in the 21st century, forcing us to ask difficult questions about our identity, epistemology, and ontology. We use the metaphor in a positive sense and do not shy away from difficult questions, such as, "How complicit are we in the system we openly criticize?" This is why the idea of partnership as a threshold concept was appealing, and we are grateful for our colleagues' critique, which forced us to expand our perspective.

\section{Kirsty Macfarlane and Jarah Dennison}

In the past, our primary responsibility was to liaise with PLAs, and to monitor the day-today running of the program - creating rosters, managing pay, and organizing the end-of-year function. The principal change in 2017 was our increased engagement with how the whole program operated, including budgets, training, managing and supervising staff, triaging support, as well as implementing new initiatives, such as workshops. The workshops that became part of the PLA program significantly increased our level of responsibility: not only were we responsible for organizing workshops and selecting appropriate PLAs to facilitate them, we were involved in discussions about workshop topics and scheduling. We also assisted in developing workshop content, and liaised with university staff.

This increased responsibility, particularly our involvement in curriculum and pedagogical decisions for workshops, pushed us outside our areas of expertise, leading to questions about our place within the university. We were neither students nor academic staff, but stranded somewhere between. Working outside our areas of expertise forced us to adapt quickly and find ways to complete tasks to a high standard. While we initially considered ourselves underqualified, compared to the academic staff who had previously completed these tasks, we personally felt capable of completing them.

Even minor mistakes, such as missing an email, engendered a sense of regret that we had let down those who had entrusted us with so much responsibility, thus reinforcing how much risk the coordinators were taking by giving us this responsibility. There were checks and balances in place to address this risk, including regular contact with the program coordinator, but we were nevertheless conscious of the significant risks involved. This put us in a high-stakes position where, although we were students, the decisions we made had significant ramifications for many others. While we quickly lost any conception of ourselves as students- 
we did not see ourselves as peers to the students we supported-neither did we view ourselves as academic staff. Undoubtedly, this position of trust and the high expectations placed on us drove us to be more professional. The responsibility pushed us to exceed our own expectations. It made us realize how much we actually had to offer to the university in terms of our experiences and expertise.

\section{ROUGH WATERS}

\section{Kirsty Macfarlane and Jarah Dennison}

Our first task, to recruit PLAs, proved to be the most challenging, even though we had done this in the past. We were instructed to nearly triple the intake of PLAs, even though Pam and Damir had told us that the program budget had been cut by $\$ 100,000$. We expressed concern that we would end up with too many PLAs and would not be able to offer them sufficient hours; in the past, PLAs had been guaranteed minimum hours and the flexibility of setting their own hours in order to balance their studies with their jobs.

This decision led to a key challenge in 2017. Our concerns proved valid, as feedback from PLAs indicated they wanted more hours. We also lost access to one of our most experienced PLAs, who took another position to cover the financial impact of her reduced roster. New PLAs were getting less experience each week, so it took longer to develop their confidence and competence.

Although we were part of a hierarchy and did not have final decision-making power, this initial experience led to tension and concerns that our views were not taken seriously. At the time we questioned whether, despite our experience with the PLA program, our status as students reduced the level of trust the leadership team had in our experience and abilities.

\section{Damir Mitric and Pam Delly}

Increasing the numbers of PLAs was imperative to meeting two institutional goals: achieving greater efficiencies, and extending the program's reach in order to prove the value of the service and ensure future funding. More PLAs would enable us to extend the program and reach more students. This was important, as the previous year's goals had not been achieved to the satisfaction of management, so our budget had been significantly reduced. Inviting our PLLs into financial discussions about the program, including recruitment, meant giving them agency in the process. As such, and to our surprise at the time, we experienced significant resistance on the issue of recruitment numbers. We understood that our directive seemed counterintuitive to Kirsty and Jarah, given their lack of experience in navigating competing strategic imperatives to achieve institutional goals, which is why we actively engaged them in a dialogue.

We were also genuinely surprised when, during the writing of this paper, Kirsty and Jarah raised this particular point as one of the main problems they experienced in 2017. While we were very sensitive to their concerns at the time, we were always explicit about the power dynamics in higher education and their influence on the decision-making process. This too was part of what we conceptualized to be a professional development opportunity for them. While the point of the partnership was to ensure that multiple perspectives, experiences, and expertise were voiced and heard, there was no escaping the reality of a highly structured and 
hierarchical chain of command. We felt it was important that they learn how their roles fit into the bigger picture, just as we understood our own roles within this hierarchy.

Hearing and reading their reflections on our decision to expand the numbersparticularly their feeling of managing the consequences of our decision-revealed that such partnerships can be at odds with the bureaucratic and corporate structures present in many universities today. This is exactly why we should engage in them. Their disruptive power forced us to reflect on our own role in a highly bureaucratized and managerial environment. Perhaps we could not escape the reality of the corporatized university, just as we could not ignore traditional ideas of teaching and learning, but we could subvert them by engaging our students as partners. In this case, simply pausing to reflect on both our treatment of each other as colleagues and the structures themselves, heavily influenced the nature of this process.

We did undervalue Kirsty and Jarah's experience and expertise by not allowing their views to influence our final decision; however, we overlooked the advice of our colleagues, rather than our students. Therefore, we were not submitting to the long-standing patriarchal model, which we were trying to change, but to strategic imperatives that had been imposed on the program. Exploring how those imperatives influence our professional everyday life and our interaction with each other has been a valuable exercise, made possible by our close partnership with Kirsty and Jarah.

\section{Kirsty Macfarlane and Jarah Dennison}

Another contested issue in 2017 was whether PLLs should be given additional access to data, and in what form. From a PLL perspective, lack of access was frustrating. For most of the year, we did not have direct access to the data collected during PLA consultations. PLAs often asked us questions that relied on us having this access, and as leaders, we sometimes felt caught in the middle. As mediators between PLAs and academic staff, we could not always satisfy the demands of both sides. Of course, we understood the hesitation; the data were confidential and privacy concerns were paramount. While we understood why we were not given access, it was of little help practically. Eventually some access was granted, proving invaluable to the management of the program by allowing greater efficiencies and effectiveness. Gaining increased access to data also improved the quality of our relationship with the leadership team, as it indicated the growing trust between us and Damir and Pam.

\section{Damir Mitric and Pam Delly}

Issues of trust and respect are highly complex and lie at the heart of successful working partnerships. Data management was an ongoing issue in our partnership, so it was not surprising to us that it emerged in Kirsty and Jarah's reflection. While we eventually made the decision to grant full access, we found this decision "troublesome, transformative, [and] irreversible" (Cook-Sather, 2014, p. 187).

To align our program with the university's strategic and business goals, we implemented much more rigorous data collection. While the PLLs were given the responsibility of collecting the data from the students who used the program, we had decided early in the partnership that Kirsty and Jarah should not have access to those data. Their requests for access became a focal point of discussions within the Learning and Teaching team, and we faced many challenges 
from our colleagues about the appropriateness of granting access to students. Clearly, some colleagues still considered the PLLs to be students, rather than colleagues. We were fully aware that the PLLs needed access to the data to do their jobs properly, but were constrained by systemic issues around data collection, confidentiality, and complications with the data platform. As such, we believed that plunging our junior colleagues into a discussion around bureaucracy and power seemed unproductive.

During our protracted discussions with other colleagues, we endeavoured to maintain an open dialogue with Kirsty and Jarah so that they were aware of the bigger-picture issues we were encountering. Our challenge, too, was how to confront a persistent culture amongst some of our colleagues that not only seemed to undervalue the professionalism of our PLLs, but also appeared to question the trust we had in them. While we felt confident in Kirsty and Jarah's professionalism and trustworthiness, it was also vital that we did not dismiss the concerns of our colleagues, which would have challenged the trust and respect already established in those collegial relationships. The protracted discussions eventually led to the agreement to allow Kirsty and Jarah access, but consensus took us most of the year.

\section{LANDING}

Navigating the rough waters of our nascent partnership has enabled us to develop a working relationship that is strong, honest, and respectful. This has been achieved by maintaining an open dialogue throughout the year in which all views could be shared, heard, and acknowledged. As with any partnership, disagreement and conflict are inevitable, and sometimes decisions are made that do not reflect a consensus. At times, this occurs because there is also no escaping the strategic imperatives that underpin a collegial partnership. However, it has been rewarding to navigate those imperatives and make decisions based on continuous and open dialogue. We will continue to face challenges as the partnership evolves, particularly as we gradually try to transform institutional culture and attitudes toward studentsas-partners. Based on our experiences of student partnership in 2017, this transformation seems much more achievable now, as we move into 2018 with a relationship that is firmly grounded in open, honest, and respectful dialogue.

\section{REFERENCES}

Cook-Sather, A. (2014). Student-faculty partnership in explorations of pedagogical practice: a threshold concept in academic development. International Journal for Academic Development, 19(3), 187-198. http://doi.org/10.1080/1360144X.2013.805694

Healey, M., Flint, A., \& Harrington, K. (2014). Engagement through partnership: students as partners in learning and teaching in higher education. York: Higher Education Academy. Retrieved from https://www.heacademy.ac.uk/system/files/resources/engagement through partnersh ip.pdf

Matthews, K. (2017). Five propositions for genuine students as partners. International Journal for Students as Partners, 1(2). Retrieved from https://mulpress.mcmaster.ca/ijsap/article/view/3315 
Mercer-Mapstone, L., Dvorakova, S. L., Matthews, K., Abbot, S., Cheng, B., Felten, P., knorr, K., Marquis, E., Shammas, R., \& Swaim, K. (2017). A systematic literature review of students as partners in higher education. International Journal for Students as Partners, 1 (1). https://doi.org/10.15173/ijsap.v1i1.3119

Meyer, J., \& Land, R. (2006). Overcoming barriers to student understanding: Threshold concepts and troublesome knowledge. London: Routledge.

Percy, A. (2014). Re-integrating academic development and academic language and learning: A call to reason. Higher Education Research \& Development, 33, (6), 1194-1207. 